\title{
REVISTA JUSTICIA Y DERECHO
}

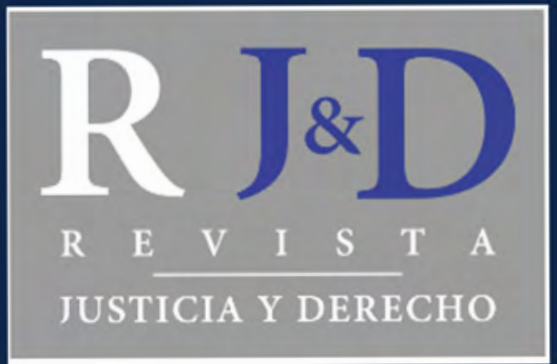

VOLUMEN 2, N. 1, 2019

ISSN 0719-9392

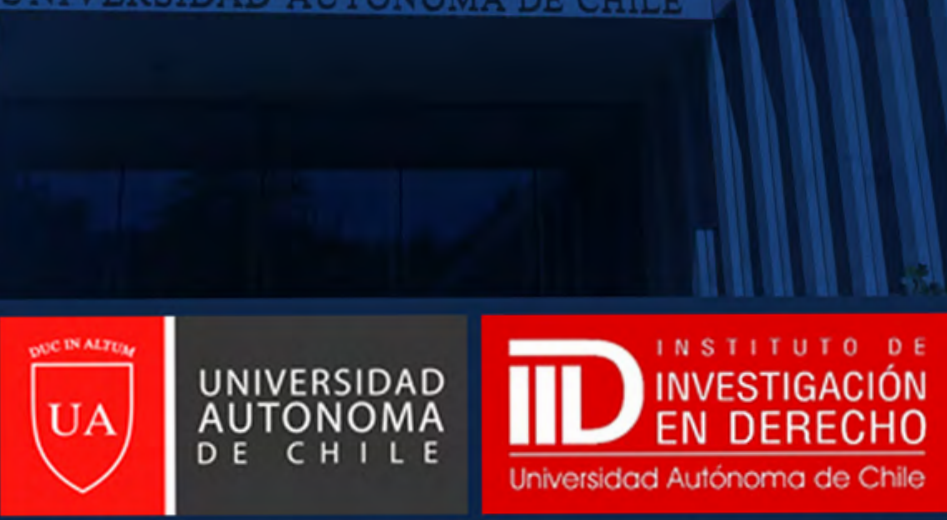

FACULTAD DE DERECHO | UNIVERSIDAD AUTÓNOMA DE CHILE 


\section{Tabla de Contenidos}

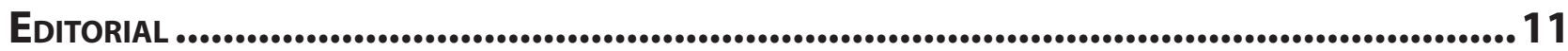
Gonzálo Ruz Lártiga

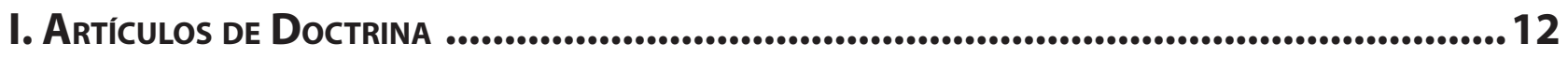

Sociedades Anónimas Deportivas como modelo de Gestión de Clubes: Viabilidad en Argentina y las experiencias en el Derecho Comparado ................................................. 14 Carlos Adolfo García

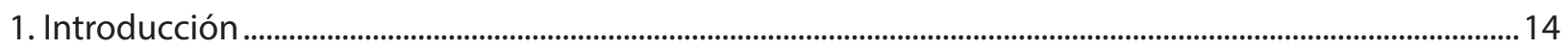

2. Deporte, estructura jurídica y negocio ....................................................................................................................... 15

3. Modelo de Sociedad Anónima Deportiva ........................................................................................................ 16

4. Antecedentes acerca de Proyectos de Sociedades Anónimas Deportivas en Argentina ......................... 16

5.- La Figura Asociativa Argentina en el Deporte y sus particularidades........................................................... 17

6. Regulación de Sociedades Anónimas Deportivas en Latinoamérica ......................................................... 18

7. Inglaterra, un modelo corporativo y profesional ................................................................................................21

8. Sociedades Anónimas Deportivas, experiencia en algunos países de Europa ..............................................23

8.1. Las Sociedades Anónimas Deportivas en España ..............................................................................................23

8.2. Modelo de Sociedad Anónima Deportiva Italiana ................................................................................................22

8.3. Francia y un enfoque Corporativo-Social de clubes .......................................................................................22

9. Franquicia en el deporte .............................................................................................................................................2 24

9.1. Estados Unidos y un modelo de gestión y negocio auténtico.....................................................................24

10. Cuestiones acerca de la implementación de la SAD .........................................................................................26

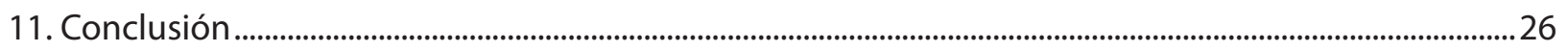

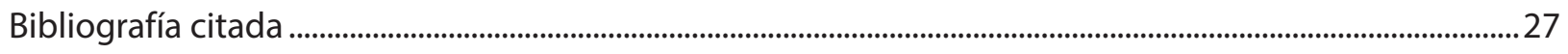

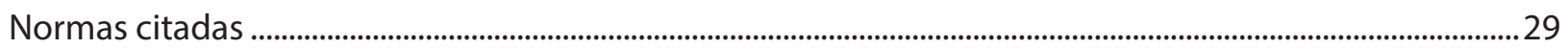

ANÁLISIS DE LA POTESTAD REGLAMENTARIA DE LAS ORDENANZAS MUNICIPALES EN EL CONTEXTO DEL ACOSO CALLEJERO: NOCIÓN, LÍMITES Y ROL EN EL ESCENARIO SOCIAL. ESTUDIO CENTRADO EN LAS ORDENANZAS MUNICIPALES DE ACOSO CALLEJERO DE LA MUNICIPALIDAD DE LAS CONDES Y DE LA MUNICIPALIDAD DE RECOLETA.................................................................................................31 Eliana Beatriz de la Merced Mallea Valverde

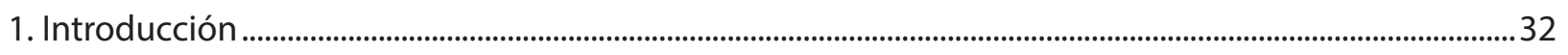

2. Ordenanza municipal como manifestación de la Potestad Reglamentaria ....................................................34

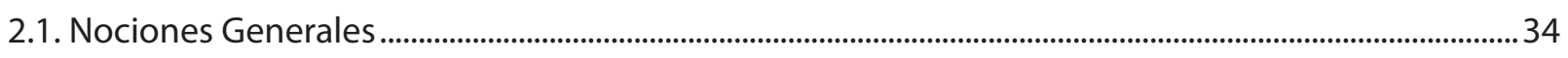

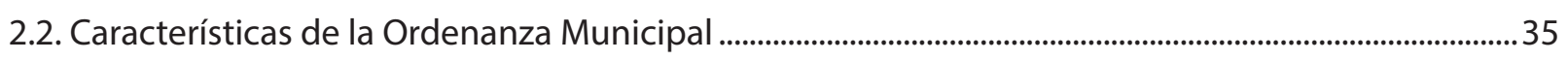

2.3. Límites de aplicación de las ordenanzas municipales ...............................................................................36 
2.3.1. Límite Material

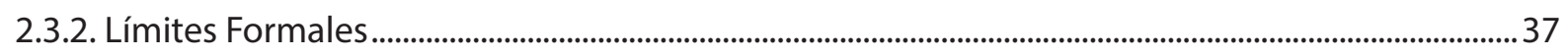

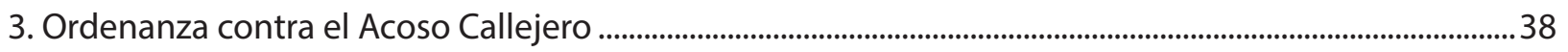

4. Análisis comparativo de los límites de las Ordenanzas Municipales de Las Condes y Recoleta............38

5. Jurisprudencia ...........................................................................................................................................................................4

5.1. Juzgados de Policía Local .......................................................................................................................................43

5.2. Pronunciamiento de la Contraloría General de la República........................................................................45

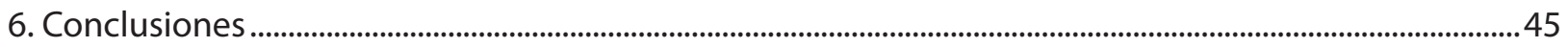

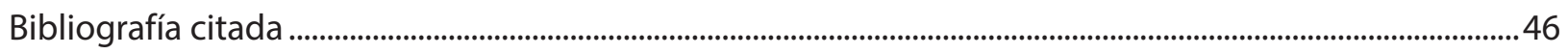

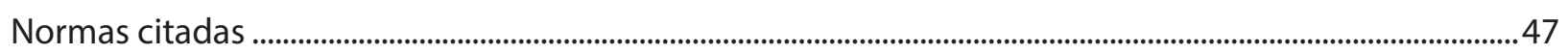

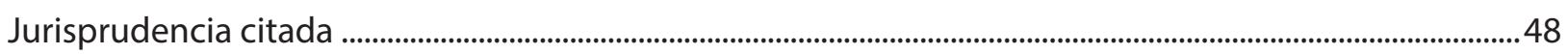

Compromiso de no repetición del Estado colombiano, en el Pos-Acuerdo 2016, una forma DE REPARACIÓN EN TRANSICIÓN, PARA LAS FARC-EP Y LAS NUEVAS Y FUTURAS VÍCTIMAS ............50 Luis Antonio Muñoz Hernández

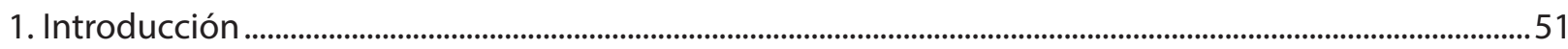

2. Desarrollo conceptual, analítico, hallazgos..............................................................................................................52

2.1. Crisis de lo colectivo en épocas de individualismo ...................................................................................53

2.2. La paz, su naturaleza y la reparación consagrada en el acuerdo ..................................................................55

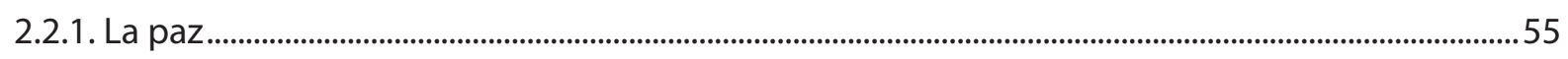

2.2.1.1. Naturaleza jurídica y constitucional de la paz en Colombia ............................................................................57

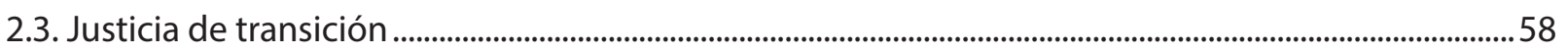

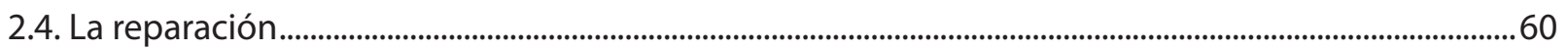

2.4.1. La reparación en el acuerdo FARC-EP - Estado colombiano (2016) .....................................................60

2.5. Las victimas del Pos-Acuerdo y su reparación. .......................................................................................................61

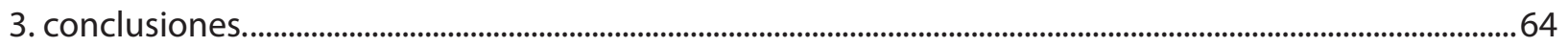

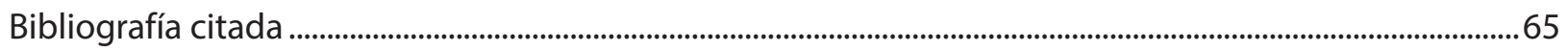

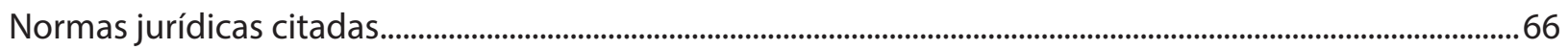

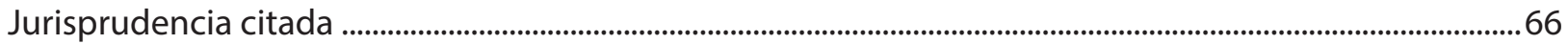

ASPECTOS REGULATORIOS DE LA PROTECCIÓN JURÍDICA DE LA PRIVACIDAD Y DE LOS DATOS PERSONALES

EN BRASIL .........................................................................................................69

Thiago Paluma y José Luiz De Moura Faleiros Júnior

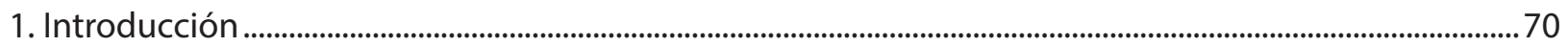

2. Relaciones jurídicas en la sociedad de la informacción..............................................................................

3. De la protección de la privacidad e de la tutela jurídica de los datos personales .......................................75

4. Los marcos regulatorios brasileños..................................................................................................................................

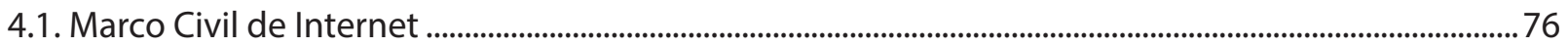




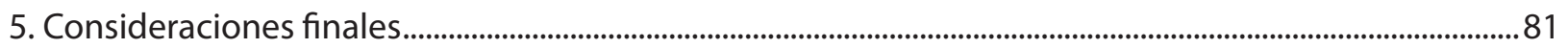

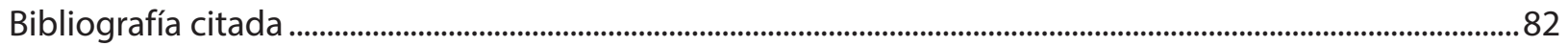

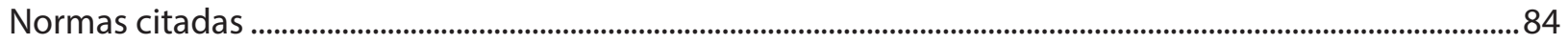

EL DOLO COMO FUENTE DE ERROR ESENCIAL ...........................................................................86

Daniel Alejandro Sánchez Carrasco

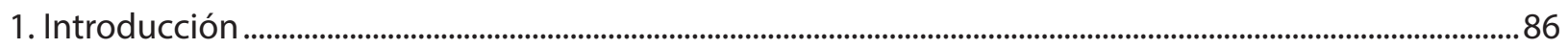

2. De la relación que existe entre el dolo y el error ..............................................................................................8 87

3. Si el dolo es fuente de error ¿Qué sucedería si este da lugar al error esencial? ............................................8 88

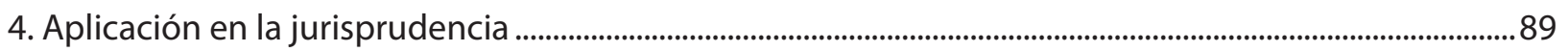

5. Consecuencias de aceptar la compatibilidad entre las acciones emanadas del dolo y el error esencial ....91

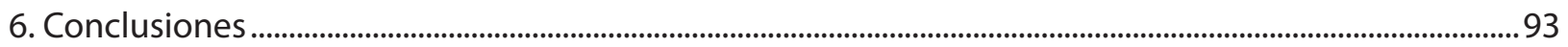

Bibliografía citada ........................................................................................................................................................ 93

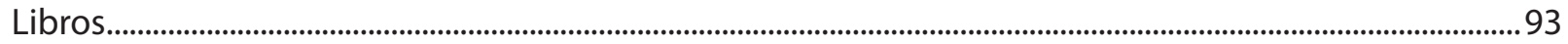

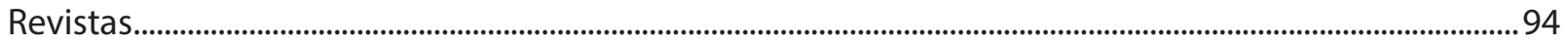

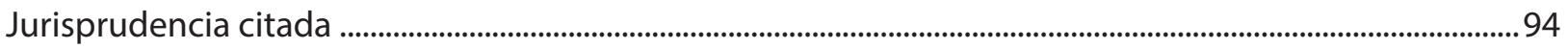

LA LEGITIMACIÓN ACTIVA EN EL EXAMEN DE ADMISIBILIDAD EN LOS PROCESOS COLECTIVOS DE CONSUMIDORES......................................................................................................96

Felipe Valenzuela Quirós y Juan Sebastián Silva Barroilhet

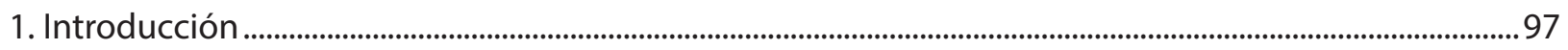

2. El examen de admisibilidad de los procesos colectivos en la legislación chilena ......................................98

2.1. El antiguo examen de admisibilidad ............................................................................................................99

2.2. El actual examen de admisibilidad.............................................................................................................101

3. La legitimación activa en la Ley de Protección de los Derechos de los Consumidores, № 19.496....101

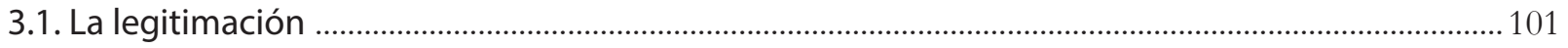

3.2. Personas legitimadas para demandar en la ley de derechos del consumidor ......................................104

4. Reflexiones constitucionales en torno a los procesos colectivos ....................................................................106

5. Conclusiones ..............................................................................................................................................................................107

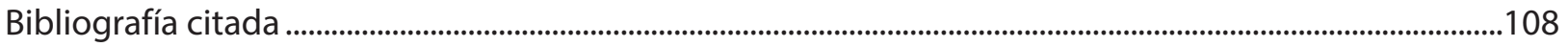

Normas citadas .................................................................................................................................................................110

Otras fuentes citadas ..........................................................................................................................................................110

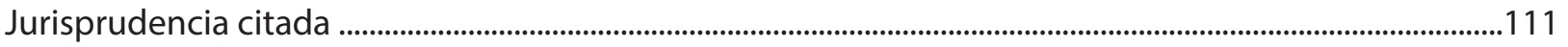




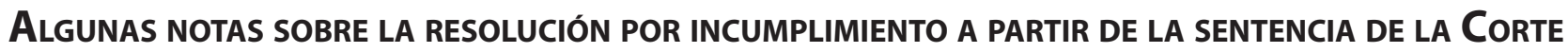
SUPREMA DE FECHA 27 DE SEPTIEMBRE DE 2016 (“INELCO CON SAESA").......................114 Branco Andrés Aravena Cuevas

1. La sentencia: hechos y resolución. ........................................................................................................................115

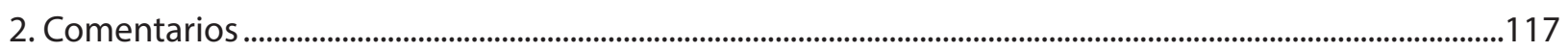

2.1. La función de la resolución por incumplimiento ..................................................................................117

2.2. El ejercicio unilateral y extrajudicial del remedio resolutorio .......................................................................119

2.3. Sobre la redacción de la cláusula del caso en comento y, en particular, lo concerniente a la entidad del incumplimiento resolutorio..............................................................................................................121

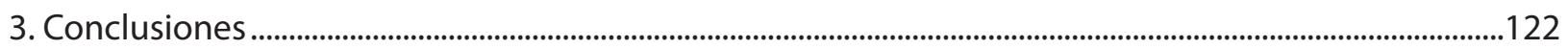

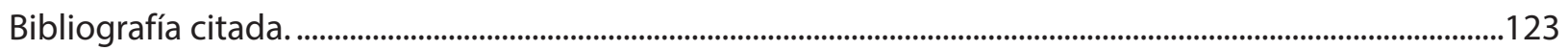

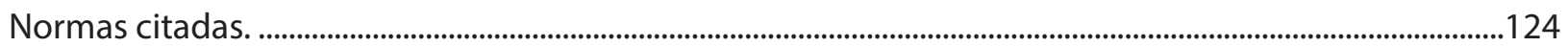

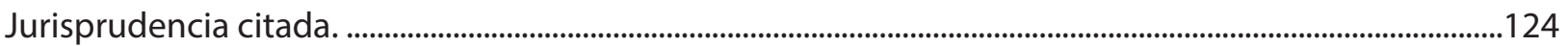

Dominancia Colectiva: Análisis de la sentencia del pasado 25 de Junio de 2018, en que la Corte Suprema acogió la reclamación deducida por la Corporación Nacional de Consumidores y Usuarios de Chile, Asociación de Consumidores en contra de la Sentencia No 154/2016 del Tribunal de Defensa de la Libre Competencia .................................126 Gabriel Budnik Ojeda

1. Hechos

2. Mercado relevante afectado y participaciones de mercado

3. Ilícito infraccional .128

4. Críticas a la sentencia de la Corte Suprema ............................................................................................130

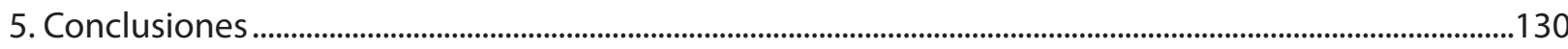

Comentario de la sentencia no. 104/2019 de 19 de febrero de 2019 de la sala de lo CiviL DEL TRIBUNAL SUPREMO ESPAÑOL: ABRE LA POSIBILIDAD DE EXTINGUIR LA PENSIÓN DE ALIMENTOS DE AQUELLOS HIJOS MAYORES DE EDAD QUE NO MANTENGAN RELACIÓN CON EL PROGENITOR OBLIGADO AL PAGO DE SU PENSIÓN DE ALIMENTOS ................................................................................. 132 Alicia Olga Castillo Saldías

1. Introducción . .133

2. Síntesis de la controversia . .133

2.1. Antecedentes de Hecho 133

2.2. Consideraciones y Fundamentos del Tribunal Supremo. . .134

3. Comentarios 135

Bibliografía citada 138 Normas citadas 
Decision C-111 of 2019: Regressions of the Constitutional Court on the matter of

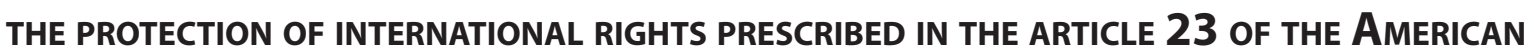
Convention on Human Rights

Juan Sebastián Villamil Rodríguez

1. Introduction

2. Decision C-028 of 2006 and the judicial dialogue with the ACHR and the United Nations Convention Against Corruption . .144

3. Decision C-111 of 2019 and the internationalization of the law in Colombia. 146

4. Conclusions 149

Courts decisions

III. ReCEnsiones

LA APLICACIÓN DEL DERECHO INTERNACIONAL POR LOS TRIBUNALES NACIONALES. RESEÑA DEL LIBRO de Díaz Fuenzalida, Juan Pablo: Principales tratados internacionales sobre derechos humanos de la ONU aplicables en los tribunales de Justicia en Chile. Concordancias CON LAS GARANTÍAS CONSTITUCIONALES CHILENAS Y JURISPRUDENCIA RELEVANTE DE LOS MÁXIMOS tribunales. Santiago, Ediciones Jurídicas de Santiago, 2019, 341 P 154 Regina Ingrid Díaz Tolosa

1. Introducción: Fundamentos de la aplicación directa del derecho internacional convencional de los derechos humanos por los tribunales chilenos.

2. Concordancias entre los órdenes internacional y nacional en materia de derechos humanos . .157

3. Jurisprudencia relevante, ejemplo de aplicación interna del derecho internacional de los derechos humanos.... . .159

4. Utilidad o usos de un libro de concordancias normativas y jurisprudencia relevante . .160

Bibliografía Citada. .. .160 